\title{
Quality of Life in Cutaneous Lupus Erythematosus
}

\author{
Rachel Klein, BA ${ }^{1,2}$, Siamak Moghadam-Kia, MD ${ }^{1,2}$, Lynne Taylor, PhD ${ }^{3}$, Christopher \\ Coley, BA ${ }^{3}$, Joyce Okawa, RN ${ }^{1,2}$, Jonathan LoMonico, BA, Mary-Margaret Chren, MD $^{4}$, and \\ Victoria P. Werth, MD $^{1,2}$ \\ ${ }^{1}$ Philadelphia VA Medical Center, Philadelphia, PA \\ ${ }^{2}$ Department of Dermatology, University of Pennsylvania School of Medicine, Philadelphia, PA \\ ${ }^{3}$ Center for Clinical Epidemiology and Biostatistics, University of Pennsylvania, Philadelphia, PA \\ ${ }^{4}$ Department of Dermatology, University of California San Francisco, San Francisco, CA
}

\section{Abstract}

\begin{abstract}
Background-Little is known about quality of life in patients with cutaneous lupus erythematosus.
\end{abstract}

Objective-We sought to determine how cutaneous lupus affects quality of life and which independent variables are associated with poor quality of life.

\begin{abstract}
Methods-157 patients with cutaneous lupus completed surveys related to quality of life, including the Skindex-29 and the SF-36.

Results-Quality of life in cutaneous lupus is severely impaired, particularly with respect to emotional well-being. Patients with cutaneous lupus have worse quality of life than those with other common dermatologic conditions, such as acne, non-melanoma skin cancer, and alopecia. With respect to mental health status, patients with cutaneous lupus have similar or worse scores than patients with hypertension, type 2 diabetes mellitus, recent myocardial infarction, and congestive heart failure. Factors related to poor quality of life include female gender, generalized disease, severe disease, distribution of lesions, and younger age.
\end{abstract}

Limitations-The study was done at a single referral-only center.

Conclusion-Patients with cutaneous lupus have very impaired quality of life, particularly from an emotional perspective.

Corresponding Author: Dr. Victoria P. Werth, Department of Dermatology, Perelman Center for Advanced Medicine, Suite 1-330A, 3400 Civic Center Boulevard, Philadelphia, PA 19104, Tel. 215-823-4208, Fax 866-755-0625, werth@ mail.med.upenn.edu.

Capsule summary:

- Cutaneous lupus erythematosuss (CLE) is associated with poor quality of life, particularly from an emotional perspective.

- Quality of life in CLEcutaneous lupus erythematosus is worse than that caused by many other dermatologic diseases and is similar to that caused by several common medical conditions.

- Factors related to poor quality of life include female gender, generalized or severe disease, young age, and distribution of lesions.

- Clinicians should address quality of life issues in high-risk patients with cutaneous lupus.

This work was presented previously in a poster session at the American College of Rheumatology's annual meeting in October 2009.

The authors declare no conflict of interest.

Publisher's Disclaimer: This is a PDF file of an unedited manuscript that has been accepted for publication. As a service to our customers we are providing this early version of the manuscript. The manuscript will undergo copyediting, typesetting, and review of the resulting proof before it is published in its final citable form. Please note that during the production process errors may be discovered which could affect the content, and all legal disclaimers that apply to the journal pertain. 


\section{Keywords}

quality of life; cutaneous lupus erythematosus; psychiatric comorbidity; Skindex-29; SF-36; CLASI

\section{Introduction}

Lupus erythematosus is a chronic autoimmune disease that frequently involves the skin. Four out of the eleven diagnostic criteria for systemic lupus erythematosus (SLE) are cutaneous in nature, and many patients have cutaneous lupus erythematosus (CLE) with little to no systemic symptoms1. The prevalence of SLE ranges from 17-48/100,000, and CLEcutaneous lupus erythematosus occurs anywhere from 1-3 times as frequently as SLE1 ${ }^{-3}$. CLE Cutaneous lupus erythematosus is categorized into three groups, including chronic (CCLE), subacute (SCLE), and acute (ACLE) forms. The most common manifestation of chronic CCLE is discoid lupus (DLE), which presents as indurated erythematous plaques and papules that can result in significant scarring and alopecia1. SCLE patients are particularly photosensitive and present with erythematous papulosquamous or annular-polycyclic plaques that tend to heal with residual postinflammatory dyspigmentation1. ACLE most often manifests as malar erythema and is frequently associated with systemic disease1. There are also a number of skin lesions that are relatively common in lupus patients but are not specific for lupus, including livedo reticularis, vasculitis, Raynaud's phenomenon, and alopecia areata. Like ACLE, these lupus nonspecific lesions are seen more commonly in those with systemic disease1.

Dermatologic diseases, in general, can have a profound influence on quality of life; they affect work, interpersonal relationships, and leisure activities4. Patients are distressed about the disease itself and how they are perceived by others as a result of their appearance 5, 6 .

Not surprisingly, there is a high proportion of psychiatric morbidity, especially anxiety and depression, in the dermatologic population, with a prevalence ranging from approximately $20-40 \%$, compared to $11-30 \%$ seen in the general population $7^{-} 14$.

Similarly, SLE has been shown to have a significant impact on quality of life. A comprehensive review by McElhone et al highlights the profound impairment in quality of life seen in patients with SLE compared to the general population15. Quality of life in SLE is similar to or worse than that in several serious medical conditions, including acquired immune deficiency syndrome, rheumatoid arthritis, and Wegener's granulomatosis15. In this review, quality of life was influenced by fatigue, social support, feelings of helplessness, coping techniques, illness related behaviors, and role strain 15 .

We hypothesized that cutaneous lupus erythematosus (CLE) in particular would have a profoundly negative effect on quality of life. Many of the manifestations, such as scarring, dyspigmentation, and alopecia, can be disfiguring, causing patients to feel very selfconscious about their appearances at home and at work. Moreover, CLE cutaneous lupus erythematosus is a chronic condition that can be managed, but not cured; thus patients are expected to see their doctors frequently and often take medications for life, many of which have potential toxicity. Even if the symptoms are controlled, photosensitive patients must strictly avoid sun exposure, which interferes with vacations and other leisure activities. As a result, many patients with cutaneous lupus erythematosusCLE patients feel trapped and burdened by their disease.

Our goal was to assess the effects of CLE on quality of life. We specifically sought to compare quality of life in CLE to that in other dermatologic and medical diseases and to 
determine independent variables associated with poor quality of life. We also compared patient and physician skin scores to assess which better correlates with quality of life. We hypothesized that quality of life would be strongly affected by cutaneous lupus erythematosusCLE relative to other dermatologic conditions and would be similar to other chronic medical diseases, as has been demonstrated with psoriasis 16, 17. We further hypothesized that the patient's skin score would better mirror quality of life than the physician's skin score.

\section{Methods}

\section{Patients}

All patients with clinical or pathologic evidence of cutaneous lupus erythematosus CLE or SLE seen in our connective-tissue disease clinic at the Hospital of the University of Pennsylvania were invited to participate in the study, regardless of whether or not they were currently undergoing treatment. 179 patients were enrolled. Of these, 157 completed all of the required questionnaires and were included in the analysis. The study was approved by our institutional review board (IRB). All patients were age 18 or above and were enrolled after signing IRB-approved informed consent and Health Insurance Portability and Accountability Act (HIPAA) forms.

\section{Questionnaires}

Each subject was asked to complete a series of questionnaires, including the Skindex-29, the SF-36, and the patient's skin score. They also answered questions regarding personal demographics and disease history. At the same visit, the physician completed the Cutaneous Lupus Erythematosus Disease Area and Severity Index (CLASI) and the physician's skin score.

\section{Skindex-29+3}

Skin-specific quality of life was measured with the previously validated Skindex-2918. This questionnaire consists of 29 items, which are used to calculate three subscales: symptoms, emotions, and functioning. The symptoms scale measures the physical burden of the disease, such as pain, itch, burning, or sensitivity. The emotions scale measures the psychological effects of the disease, such as depression, anxiety, embarrassment, or anger. The functioning subscale focuses on the changes to daily life, such as work, sleep, and relationships with others. We added a fourth subscale, consisting of three questions, to assess lupus-specific issues, such as photosensitivity and alopecia. Each question and subscale range from 0-100 points, with higher scores indicating worse quality of life. Scores in cutaneous lupus erythematosus were compared to other dermatologic diseases (unpublished data obtained either by our group or by personal communication with Dr. Mary-Margaret Chren).

\section{SF-36}

Quality of life related to general health was measured with the previously validated SF-3619, 20. This questionnaire consists of 36 items, which are used to calculate eight subscales: physical functioning, (PF), role-physical (RP), bodily pain (BP), general health $(\mathrm{GH})$, vitality (VT), social functioning (SF), role-emotional (RE), and mental health (MH). Physical $\mathrm{F}$ addresses physical activities associated with daily life, such as bathing, walking, or carrying groceries. Role-Pphysical assesses how physical health affects work. BodilyP measures pain severity and how it interferes with daily activities. General Hhealth describes how the patient perceives his health status. VTitality assesses how energetic or tired the subject feels. SFocial functioning measures how much emotional or physical problems have interfered with usual social activities. Role-Eemotional addresses how the subject's 
emotional state has influenced work and other daily activities. Finally, MH mental health assesses the subject's mood, specifically focusing on feelings of sadness and anxiety. Scores range from $0-100$, with higher scores indicating better quality of life. In this analysis, normbased scores were used, which were based on a mean of 50 and standard deviation of 10 for the U.S. general population.

\section{Physician and patient skin scores}

At each visit, both the physician and patient were asked to rate the severity of the patient's skin disease on a scale from 0-10, with higher scores indicating less severe skin disease.

\section{CLASI}

The CLASI is a validated tool that is completed by the physician, which quantifies disease severity in cutaneous lupus erythematosusCLE21²3. It measures both activity (erythema and scale) and damage (dyspigmentation and scarring), with additional points granted for each area of involved skin. Activity scores range from 0-70, and damage scores range from $0-56$. Higher scores are indicative of more severe skin disease. Severity groups are indicated by the following CLASI activity score ranges: mild (0-9), moderate (10-20), and severe (21-70).

\section{Statistical analysis}

Overall quality of life in cutaneous lupus erythematosusCLE was assessed by looking at summary statistics for the Skindex-29+3 and examining inter-correlations amongst subscales. Quality of life in cutaneous lupus erythematosusCLE was compared to that in other dermatologic conditions by comparing means for the three primary Skindex-29 subscales (symptoms, emotions, and functioning) in cutaneous lupus erythematosusCLE to those in nine eight other cutaneous skin diseases, as well as to those without skin disease (unpublished data obtained either by our group or by personal communication with Dr. Mary-Margaret Chren) (means for these nine diseases were provided by Chren MM). Quality of life in cutaneous lupus erythematosusCLE was similarly compared to that in five common medical conditions, as well as the general population, using norm-based scores for the eight subscales of the SF-3624. In each analysis, means were compared for each groups using a two-tailed, one sample t-test. To minimize the experiment-wise error rate, the t-test comparisons for each subscale were evaluated with statistical significance designated as $\mathrm{p}<0.01$.

We also examined the relationship between quality of life (a dependent variable based on Skindex-29+3 scores) and several independent variables, including gender, race, disease subtype, severity, lesion distribution, age, and disease duration. We hypothesized that these independent variables might be associated with poor quality of life based on previous reports in the literature and our subjective experiences with patients. Associations with quality of life were tested using t-tests and Wilcoxon tests (two-sample, two-sided); ANOVA (using GLM) and LSD post hoc comparison at $\mathrm{p}<0.05$; and correlations (Spearman and Pearson). The use of parametric versus non-parametric tests was dependent on the scale of measurement and distribution of the results. Thus, Pearson's correlation coefficients were used when the distribution of scores was unimodal and symmetrical. Thus, Spearman's correlation coefficients were used when the distribution of scores was not symmetrical.

Finally, to examine which skin score (patient or physician) better correlates with quality of life (based on Skindex-29+3 subscales) we computed correlations with their 95\% CI. All calculations were done using SAS 9.1. 


\section{Results}

\section{Patient characteristics}

157 patients were included in the analysis. The majority of the participants were female $(83 \%)$ or Caucasian $(68 \%)$. A variety of cutaneous lupus erythematosusCLE subtypes were represented, including SCLE (24\%), localized DLE (23\%), generalized DLE (15\%), tumid lupus (8\%), ACLE (5\%), lupus panniculitis (3\%), and lupus non-specific skin disease (8\%). Most of the patients enrolled had mild disease (68\%), although there were a number with moderate $(20 \%)$ and severe disease $(13 \%)$ as well. Participants were taking a range of different classes of medications, including topical steroids (17\%), prednisone (23\%), antimalarials (59\%), immunosuppressives (22\%), and thalidomide (3\%), while some were untreated $(26 \%)$ (Table 1).

\section{General overview of quality of life in cutaneous lupus erythematosusCLE}

Of all of the Skindex-29+3 subscales, patients with cutaneous lupus erythematosusCLE patients were most affected in the lupus-specific and emotions domains, which had a mean (SD) of 57(28) and 48(28). They were least affected in the functioning domain [28 (25)] (Table 2). Within the lupus-specific domain, patients were most concerned about spending time outdoors [62 (33)] and losing hair [56 (37)]. Within the emotions domain, patients were most concerned about their skin getting worse [65 (30)] and that their condition might be serious [56 (30)]. They also expressed frustration [55 (33)] and annoyance [54 (33)]. Within the emotions domain, they were least concerned with their disease being a problem for their loved ones [15 (24)] and interfering with their sex lives [17 (28)] (Figure 1). The subscales were highly intercorrelated, such that a high score in one tended to be associated with high scores in the others $\left(r_{p}=0.37-0.67, r_{s p}=0.56-0.81\right.$, all $\left.\mathrm{p}<0.0001\right)$.

\section{Cutaneous lupus erythematosus CLE vs. other skin diseases}

Skindex-29 scores in cutaneous lupus erythematosusCLE were compared to those in eight other dermatologic conditions, and to those in patients without skin disease. The lupus population was among the most severely affected in the emotions domain [48 (28)], similar to the population of patients with dermatomyositis [45 (27)] and vulvodynia [50 (20)], with all other disease populations having significantly lower scores (all $\mathrm{p}<0.0009$ ). CLE pPatients with cutaneous lupus erythematosus were also profoundly impacted in the functioning domain [28 (25)] compared to patients with other conditions. The symptom burden [40 (23)] in cutaneous lupus erythematosusCLE was similar to dermatomyositis [42 (25)] and psoriasis [42 (21)] and was only less than vulvodynia [50 (17)] and eczema [48 (23)]. Across all Skindex-29 subscales, quality of life in CLEcutaneous lupus erythematosus was significantly worse than patients without skin disease (all $\mathrm{p}<0.0001)$ (Table 2).

\section{Cutaneous lupus erythematosusCLE vs. other medical conditions}

SF-36 scores in cutaneous lupus erythematosusCLE were compared to five common chronic medical conditions, including hypertension, congestive heart failure (CHF), type 2 diabetes mellitus, recent myocardial infarction, and clinical depression. With respect to the subscales related to mental health (VTvitality, SFsocial functioning, RE, MH)mental health), patients with cutaneous lupus erythematosusCLE were similar to patients with CHF and worse than patients with hypertension, diabetes, and a recent myocardial infarction (all $\mathrm{p} \leq 0.01$, excluding RE role-emotional for recent MImyocardial infarction). With respect to the subscales related to physical health (physical functioning, role-physical, bodily pain, general health), patients with cutaneous lupus erythematosus were similar to those with hypertension and diabetes. Patients with cutaneous lupus erythematosus were similar to those with clinical depression in terms of physical functioning and perceived general health. With the exception 
of bodily pain, patients with cutaneous lupus erythematosus had significantly worse quality of life than the general population across all subscales (all $p<0.01$ ) (Table 3 ).

\section{Factors related to quality of life}

A number of factors were tested for an independent association with poor quality of life including gender, ethnicity, disease subtype, disease severity, distribution of lesions, disease duration, and current age. Female gender was associated with poor quality of life in all three Skindex domains and in the lupus-specific domain (all $\mathrm{p}<0.006$ ); however there was no significant difference in quality of life amongst different ethnicities (Figure 2a,b). Generalized disease (DLE and SCLE) was associated with impaired functioning compared to localized DLE (all p<0.05), however disease subtype did not have a significant impact on other aspects of quality of life (Figure 2c). Increased disease severity correlated with worse quality of life for all three subscales and the lupus-specific subscale $\left(r_{\mathrm{sp}}=0.24-0.36\right.$, all $\mathrm{p}<0.003$ ). Significant differences between severity groups were seen in the symptoms (mild vs. moderate vs. severe), emotions (mild vs. severe), functioning (mild and moderate vs. severe), and lupus-specific (mild vs. moderate) domains (all $\mathrm{p}<0.05$ ) (Figure 2d).

With respect to distribution of lesions, there was a correlation between the presence of at least one facial lesion and worse quality of life across the symptoms $\left(r_{p}=0.24, p=0.0029\right)$, functioning $\left(\mathrm{r}_{\mathrm{sp}}=0.24, \mathrm{p}=0.0035\right)$, and emotions $\left(\mathrm{r}_{\mathrm{p}}=0.23, \mathrm{p}=0.0058\right)$ subscales. There was also a correlation between the presence of either inflammatory alopecia or mucous membrane lesions with worse symptoms and lupus-specific scores $\left(r_{p}=0.16-0.26\right.$, all $\mathrm{p}<0.05$ ). Younger age was correlated with more symptomatic and emotional impairment $\left(r_{p}=0.16-0.22\right.$, all $\left.p<0.04\right)$, however there was no significant correlation between quality of life and disease duration (Table 4).

\section{Correlation between physician and patient skin scores and quality of life}

To assess if the physician or patient skin scores were related to quality of life, correlation coefficients for each score and the Skindex-29 were calculated. The mean patient skin score was lower than the mean physician skin score (4.86 vs. 7.52, respectively). However, the two scores had similar correlations with quality of life across all four subscales (Table 5).

\section{Discussion}

These results indicate that patients with cutaneous lupus erythematosus have very poor quality of life, particularly with respect to emotions, photosensitivity, and hair loss. When compared to a number of other skin diseases, patients with cutaneous lupus erythematosus are amongst the most severely affected by their disease. With respect to common medical conditions, the psychological aspects of quality of life in cutaneous lupus erythematosus are similar to or worse than those in chronic hypertension, congestive heart failure, type 2 diabetes, and a recent myocardial infarction.

Female gender was strongly associated with poor quality of life in lupus, which is consistent with reports in the literature showing increased psychiatric comorbidity and worse quality of life in women with acne, pemphigus, cutaneous lymphoma, vitiligo, psoriasis, and chronic urticaria $7,25^{-} 30$. Increased disease severity was also correlated with poor quality of life in lupus, as has been demonstrated in acne, psoriasis, cutaneous lymphoma, and vitiligo 7 , $25^{-} 28.7,9^{-} 11,26,27,29$. The impaired functioning seen in generalized disease is not surprising, given the increased burden of disease in these patients compared to those with localized disease only.

Reports in the literature with respect to an association between distribution of lesions and psychiatric comorbidity are conflicting $8^{-} 11$. In this study, there was a clear connection 
between facial lesions and impaired quality of life, which is understandable given the conspicuous nature of such lesions. Inflammatory alopecia was correlated with higher symptoms and lupus-specific scores; this likely reflects the itch associated with inflammatory alopecia and the patients' fear of losing more hair. This finding is consistent with previous reports demonstrating worse quality of life in lupus patients with alopecia compared to those without alopecia31, 32. Unlike previous reports indicating no connection between psychiatric disease and current age or duration of disease $7,10,11,31$, our results suggest a small correlation between poor quality of life and younger age.

We hypothesized that the patient's skin score would better reflect quality of life because it indicates the patient's subjective perception of his disease, which does not necessarily mirror objective disease severity. However, these results indicate that the physician's skin score correlates equally well with quality of life. This, together with the correlation between quality of life and CLASI scores, indicates that the physician can recognize patients at risk for impaired quality of life based on assessments of disease severity.

Addressing quality of life issues is critical when treating patients with skin disease. The high prevalence of depression and anxiety in the dermatologic population may be in part due to poor quality of life, which is strongly linked to psychiatric comorbidity. In fact, quality of life predicts psychiatric well-being better than clinical severity, the end-point most physicians use when treating patients 7,33 . Moreover, there is a high rate of suicidal ideation amongst dermatology patients, particularly those with high Skindex scores, ranging from 5.6-8.6\%, compared to only 2.4-3.3\% seen in the general medical population 34, 35 . Psychiatric disease in turn is associated with poor compliance and increased perception of symptoms $33,36,37$. Thus better recognition of this problem may help alleviate both the mental and physical burdens of the disease.

Unfortunately, dermatologists do a poor job of identifying psychiatric disease in their patients (detection sensitivity 33\%)38. Therefore, when examining a patient who is at high risk for poor quality of life, it would be reasonable to screen for psychiatric distress and suicidal ideation. Those with mild impairments should be encouraged to discuss their concerns at clinic visits or in a support-group setting. Many find it reassuring to have their feelings validated and to learn that others with cutaneous lupus have similar concerns. Those with more severe impairments resulting in psychiatric disease and suicidal ideation should be referred to psychiatry as needed. For all patients, it may not be sufficient to simply treat according to disease severity; patients with seriously impaired quality of life may benefit from a change in therapy even if their skin disease is relatively mild.

Of note, all of the subjects included in this study are patients treated at the connective-tissue disease clinic at the Hospital of the University of Pennsylvania, which is a referral-only center. As such, these patients may have more severe or refractory disease than those in the general cutaneous lupus erythematosus population, who are managed by general dermatologists. Moreover, high Skindex-29 scores were considered predictive of psychiatric comorbidity, however subjects were not formally screened for psychiatric disease with questionnaires like the GHQ-12. In addition, the cross-sectional nature of this study made it difficult to ascertain cause-and-effect in understanding the deterioration of quality of life. An earlier study done by some members of our group demonstrated a weak correlation between changes in disease severity, as measured by the CLASI, and changes in quality of life, as measured by the Skindex-2939. However, this study was limited by the small sample size $(\mathrm{N}=8)$. Therefore, larger studies must be done to elucidate how quality of life changes over time and whether or not it improves with treatment. 
In conclusion, this study indicates that patients with cutaneous lupus erythematosus suffer from poor quality of life, which profoundly impacts their overall health and sense of wellbeing. As such, it is an issue that should be acknowledged and managed.

\section{Acknowledgments}

Funding sources: This material is based upon work supported by the National Institutes of Health, including NIH K24-AR 02207 (Werth), NIH K24 AR052667 (Chren), and training grant NIH T32-AR007465-25 (Klein). This work was also partially supported by a Merit Review Grant from the Department of Veterans Affairs Veterans Health Administration, Office of Research and Development, Biomedical Laboratory Research and Development.

\section{References}

1. Werth VP. Clinical manifestations of cutaneous lupus erythematosus. Autoimmun Rev. 2005; 4:296-302. [PubMed: 15990077]

2. Durosaro O, Davis MD, Reed KB, Rohlinger AL. Incidence of cutaneous lupus erythematosus, 1965-2005: a population-based study. Arch Dermatol. 2009; 145:249-53. [PubMed: 19289752]

3. Tebbe B, Orfanos CE. Epidemiology and socioeconomic impact of skin disease in lupus erythematosus. Lupus. 1997; 6:96-104. [PubMed: 9061657]

4. Finlay AY. Quality of life assessments in dermatology. Semin Cutan Med Surg. 1998; 17:291-6. [PubMed: 9859917]

5. Richards HL, Fortune DG, Griffiths CE, Main CJ. The contribution of perceptions of stigmatisation to disability in patients with psoriasis. J Psychosom Res. 2001; 50:11-5. [PubMed: 11259795]

6. Fortune DG, Main CJ, O'Sullivan TM, Griffiths CE. Quality of life in patients with psoriasis: the contribution of clinical variables and psoriasis-specific stress. Br J Dermatol. 1997; 137:755-60. [PubMed: 9415236]

7. Picardi A, Abeni D, Melchi CF, Puddu P, Pasquini P. Psychiatric morbidity in dermatological outpatients: an issue to be recognized. Br J Dermatol. 2000; 143:983-91. [PubMed: 11069507]

8. Hughes JE, Barraclough BM, Hamblin LG, White JE. Psychiatric symptoms in dermatology patients. Br J Psychiatry. 1983; 143:51-4. [PubMed: 6882992]

9. Wessely SC, Lewis GH. The classification of psychiatric morbidity in attenders at a dermatology clinic. Br J Psychiatry. 1989; 155:686-91. [PubMed: 2611599]

10. Woodruff PW, Higgins EM, du Vivier AW, Wessely S. Psychiatric illness in patients referred to a dermatology-psychiatry clinic. Gen Hosp Psychiatry. 1997; 19:29-35. [PubMed: 9034809]

11. Aktan S, Ozmen E, Sanli B. Psychiatric disorders in patients attending a dermatology outpatient clinic. Dermatology. 1998; 197:230-4. [PubMed: 9812026]

12. Attah Johnson FY, Mostaghimi H. Co-morbidity between dermatologic diseases and psychiatric disorders in Papua New Guinea. Int J Dermatol. 1995; 34:244-8. [PubMed: 7790138]

13. Picardi A, Pasquini P, Abeni D, Fassone G, Mazzotti E, Fava GA. Psychosomatic assessment of skin diseases in clinical practice. Psychother Psychosom. 2005; 74:315-22. [PubMed: 16088270]

14. Kessler RC, McGonagle KA, Zhao S, Nelson CB, Hughes M, Eshleman S, et al. Lifetime and 12month prevalence of DSM-III-R psychiatric disorders in the United States. Results from the National Comorbidity Survey. Arch Gen Psychiatry. 1994; 51:8-19. [PubMed: 8279933]

15. McElhone K, Abbott J, Teh LS. A review of health related quality of life in systemic lupus erythematosus. Lupus. 2006; 15:633-43. [PubMed: 17120589]

16. Rapp SR, Feldman SR, Exum ML, Fleischer AB Jr, Reboussin DM. Psoriasis causes as much disability as other major medical diseases. J Am Acad Dermatol. 1999; 41:401-7. [PubMed: 10459113]

17. Sampogna F, Tabolli S, Soderfeldt B, Axtelius B, Aparo U, Abeni D. Measuring quality of life of patients with different clinical types of psoriasis using the SF-36. Br J Dermatol. 2006; 154:844-9. [PubMed: 16634884]

18. Chren MM, Lasek RJ, Flocke SA, Zyzanski SJ. Improved discriminative and evaluative capability of a refined version of Skindex, a quality-of-life instrument for patients with skin diseases. Arch Dermatol. 1997; 133:1433-40. [PubMed: 9371029] 
19. Ware JE Jr, Sherbourne CD. The MOS 36-item short-form health survey (SF-36). I. Conceptual framework and item selection. Med Care. 1992; 30:473-83. [PubMed: 1593914]

20. McHorney CA, Ware JE Jr, Raczek AE. The MOS 36-Item Short-Form Health Survey (SF-36): II. Psychometric and clinical tests of validity in measuring physical and mental health constructs. Med Care. 1993; 31:247-63. [PubMed: 8450681]

21. Albrecht J, Taylor L, Berlin JA, Dulay S, Ang G, Fakharzadeh S, et al. The CLASI (Cutaneous Lupus Erythematosus Disease Area and Severity Index): an outcome instrument for cutaneous lupus erythematosus. J Invest Dermatol. 2005; 125:889-94. [PubMed: 16297185]

22. Albrecht J, Werth VP. Development of the CLASI as an outcome instrument for cutaneous lupus erythematosus. Dermatol Ther. 2007; 20:93-101. [PubMed: 17537137]

23. Bonilla-Martinez ZL, Albrecht J, Troxel AB, Taylor L, Okawa J, Dulay S, et al. The cutaneous lupus erythematosus disease area and severity index: a responsive instrument to measure activity and damage in patients with cutaneous lupus erythematosus. Arch Dermatol. 2008; 144:173-80. [PubMed: 18283174]

24. Ware, JE., Jr; Kosinski, M.; Gandek, B. SF-36 Health Survey: Manual \& Interpretation Guide. Lincoln, RI: QualityMetric Incorporated; 2005.

25. Jones-Caballero M, Chren MM, Soler B, Pedrosa E, Penas PF. Quality of life in mild to moderate acne: relationship to clinical severity and factors influencing change with treatment. J Eur Acad Dermatol Venereol. 2007; 21:219-26. [PubMed: 17243958]

26. Paradisi A, Sampogna F, Di Pietro C, Cianchini G, Didona B, Ferri R, et al. Quality-of-life assessment in patients with pemphigus using a minimum set of evaluation tools. J Am Acad Dermatol. 2009; 60:261-9. [PubMed: 19004524]

27. Sampogna F, Frontani M, Baliva G, Lombardo GA, Alvetreti G, Di Pietro C, et al. Quality of life and psychological distress in patients with cutaneous lymphoma. Br J Dermatol. 2009; 160:81522. [PubMed: 19120325]

28. Sampogna F, Raskovic D, Guerra L, Pedicelli C, Tabolli S, Leoni L, et al. Identification of categories at risk for high quality of life impairment in patients with vitiligo. Br J Dermatol. 2008; 159:351-9. [PubMed: 18565189]

29. Sampogna F, Tabolli S, Mastroeni S, Di Pietro C, Fortes C, Abeni D. Quality of life impairment and psychological distress in elderly patients with psoriasis. Dermatology. 2007; 215:341-7. [PubMed: 17911993]

30. Maurer M, Ortonne JP, Zuberbier T. Chronic urticaria: a patient survey on quality-of-life, treatment usage and doctor-patient relation. Allergy. 2009; 64:581-8. [PubMed: 19175591]

31. Ferraz LB, Almeida FA, Vasconcellos MR, Faccina AS, Ciconelli RM, Ferraz MB. The impact of lupus erythematosus cutaneous on the Quality of life: the Brazilian-Portuguese version of DLQI. Qual Life Res. 2006; 15:565-70. [PubMed: 16547795]

32. Ferraz LB, de Almeida FA, Vasconcellos MR, Ferraz MB. Alopecia impairs the quality of life of patients with lupus erythematosus. Arch Dermatol. 2006; 142:110. [PubMed: 16415400]

33. Sampogna F, Picardi A, Chren MM, Melchi CF, Pasquini P, Masini C, et al. Association between poorer quality of life and psychiatric morbidity in patients with different dermatological conditions. Psychosom Med. 2004; 66:620-4. [PubMed: 15272112]

34. Gupta MA, Gupta AK. Depression and suicidal ideation in dermatology patients with acne, alopecia areata, atopic dermatitis and psoriasis. Br J Dermatol. 1998; 139:846-50. [PubMed: 9892952]

35. Picardi A, Mazzotti E, Pasquini P. Prevalence and correlates of suicidal ideation among patients with skin disease. J Am Acad Dermatol. 2006; 54:420-6. [PubMed: 16488292]

36. Gupta MA, Gupta AK, Schork NJ, Ellis CN. Depression modulates pruritus perception: a study of pruritus in psoriasis, atopic dermatitis, and chronic idiopathic urticaria. Psychosom Med. 1994; 56:36-40. [PubMed: 8197313]

37. Renzi C, Picardi A, Abeni D, Agostini E, Baliva G, Pasquini P, et al. Association of dissatisfaction with care and psychiatric morbidity with poor treatment compliance. Arch Dermatol. 2002; 138:337-42. [PubMed: 11902984] 
38. Picardi A, Amerio P, Baliva G, Barbieri C, Teofoli P, Bolli S, et al. Recognition of depressive and anxiety disorders in dermatological outpatients. Acta Derm Venereol. 2004; 84:213-7. [PubMed: 15202838]

39. Gaines E, Bonilla-Martinez Z, Albrecht J, Taylor L, Okawa J, Troxel AB, et al. Quality of life and disease severity in a cutaneous lupus erythematosus pilot study. Arch Dermatol. 2008; 144:10612. [PubMed: 18711088]

\section{Abbreviations and acronyms}

ACLE Acute cutaneous lupus erythematosus

BP bodily pain

CCLE chronic cutaneous lupus erythematosus

CHF congestive heart failure

CLASI Cutaneous Lupus Erythematosus Disease Area and Severity Index

DLE discoid lupus erythematosus

DMII Type 2 diabetes

GH general health

HIPAA Health Insurance Portability and Accountability Act

HTN hypertension

IRB institutional review board

MH mental health

MI Myocardial infarction

PF physical functioning

RE role-emotional

RP role-physical

SF social functioning

SCLE subacute cutaneous lupus erythematosus

SLE systemic lupus erythematosus

VT vitality 


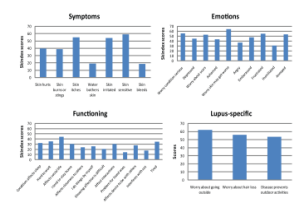

Figure 1. Details of quality of life in lupus erythematosus

The mean scores for individual questions within each subscore and the lupus-specific score were calculated. 


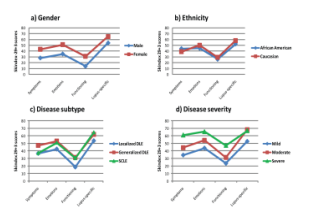

Figure 2. Factors related to quality of life I

Mean Skindex-29+3 scores are given for a) genders b) ethnicities c) disease subtypes and d) disease severity. 


\section{Patient Characteristics}

Table 1

Five patients were excluded from the severity analysis because CLASI scores were not calculated on the initial visit. For the medication summary, subjects that were taking multiple medications were counted more than once. Abbreviations used: discoid lupus erythematosus (DLE), subacute cutaneous lupus erythematosus (SCLE), and acute cutaneous lupus erythematosus (ACLE).

\begin{tabular}{|c|c|c|c|}
\hline & & $\mathbf{N}$ & $\%$ \\
\hline \multirow[t]{2}{*}{ Gender } & Male & 27 & 17 \\
\hline & Female & 130 & 83 \\
\hline \multirow[t]{4}{*}{ Ethnicity } & Caucasian & 107 & 68 \\
\hline & African-American & 41 & 26 \\
\hline & Asian & 7 & 4 \\
\hline & Hispanic/Latino & 2 & 1 \\
\hline Age & & 157 & 47 (mean) \\
\hline \multirow[t]{9}{*}{ Lupus subtype } & Generalized DLE & 23 & 15 \\
\hline & Localized DLE & 36 & 23 \\
\hline & Tumid & 12 & 8 \\
\hline & Panniculitis & 5 & 3 \\
\hline & SCLE & 38 & 24 \\
\hline & ACLE & 8 & 5 \\
\hline & Lupus non-specific & 13 & 8 \\
\hline & Other & 11 & 7 \\
\hline & Multiple subtypes & 11 & 7 \\
\hline \multirow[t]{3}{*}{ Disease severity } & Mild & 103 & 68 \\
\hline & Moderate & 30 & 20 \\
\hline & Severe & 19 & 13 \\
\hline \multirow[t]{7}{*}{ Current therapy } & Topical steroids & 27 & 17 \\
\hline & Antimalarial & 93 & 59 \\
\hline & Immunosuppressive & 34 & 22 \\
\hline & Thalidomide & 4 & 3 \\
\hline & Prednisone & 36 & 23 \\
\hline & Other & 14 & 9 \\
\hline & No therapy & 41 & 26 \\
\hline
\end{tabular}




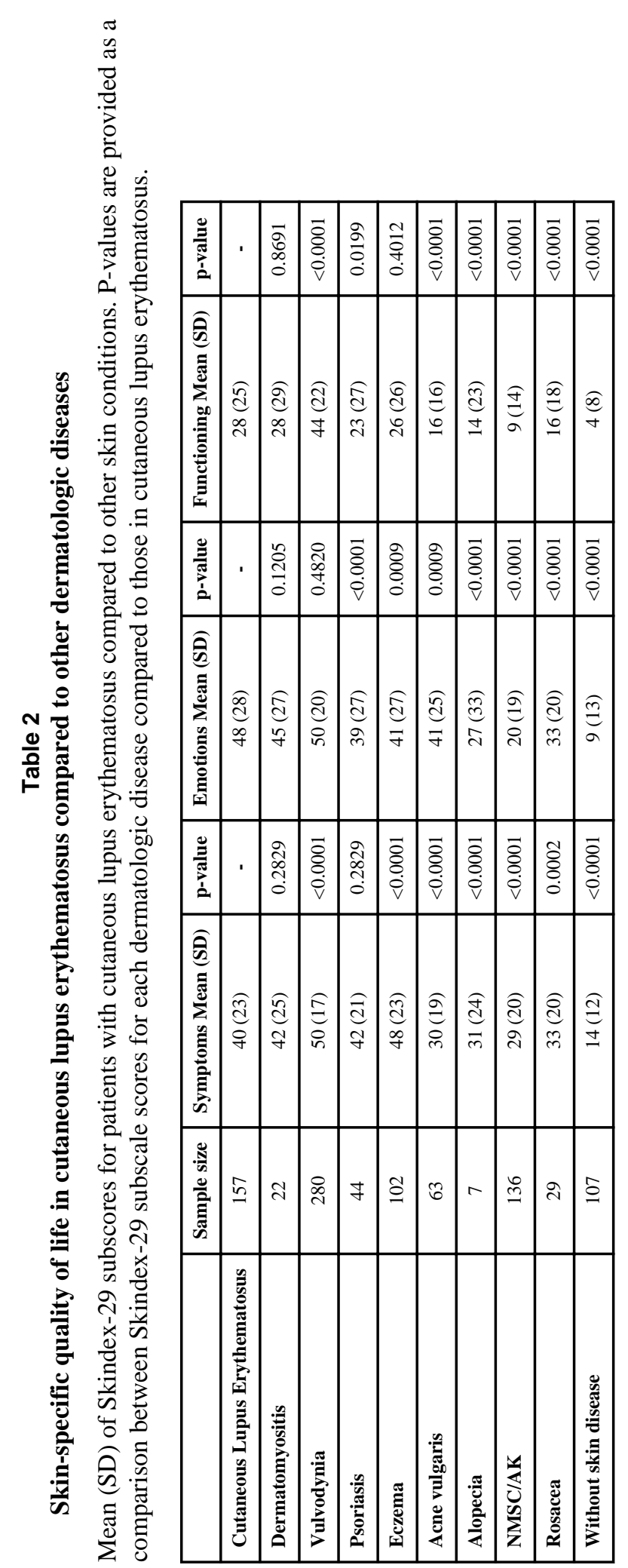

J Am Acad Dermatol. Author manuscript; available in PMC 2012 May 1. 


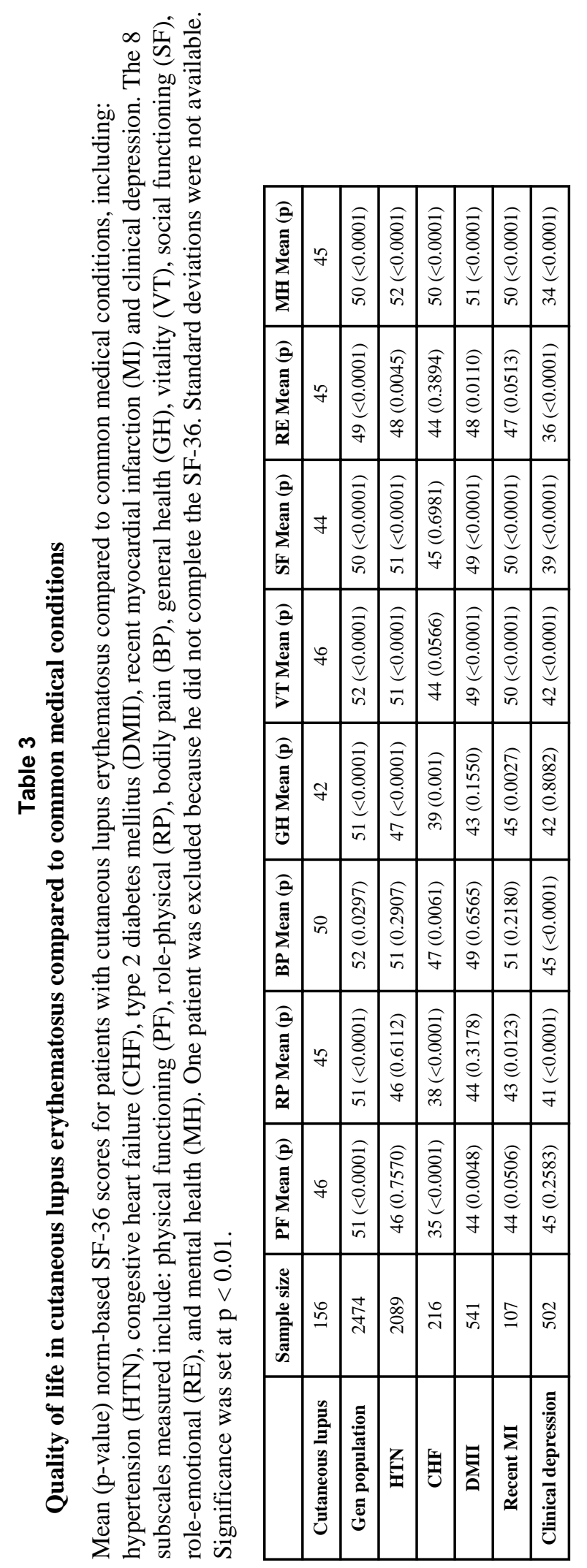

J Am Acad Dermatol. Author manuscript; available in PMC 2012 May 1. 


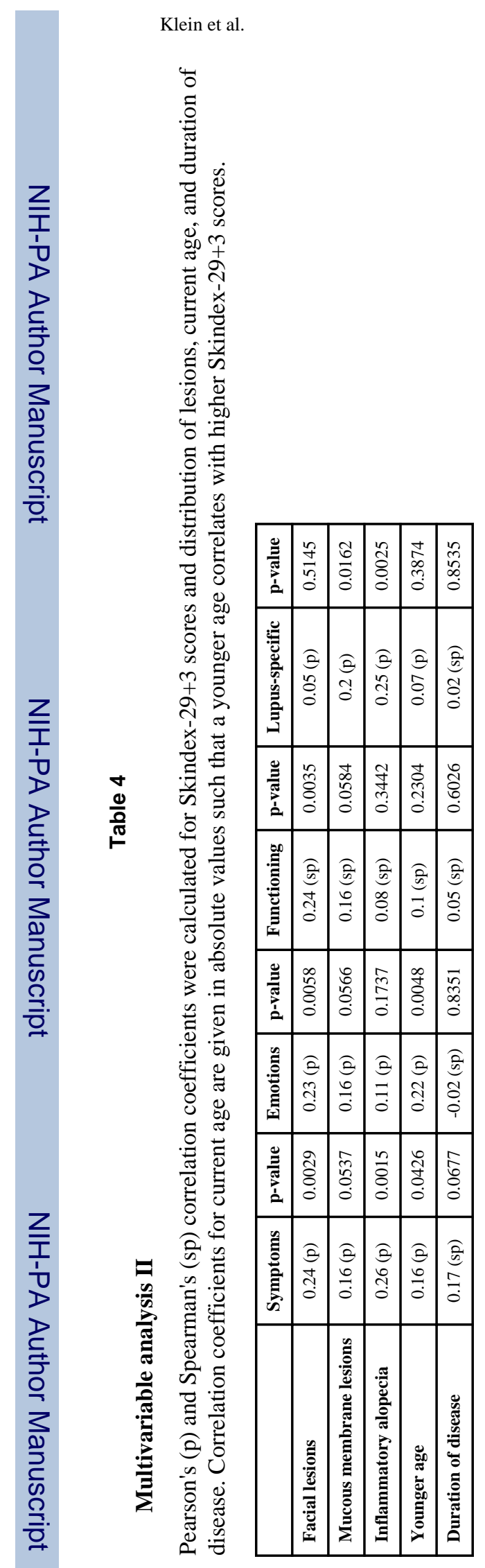

Page 16

J Am Acad Dermatol. Author manuscript; available in PMC 2012 May 1. 


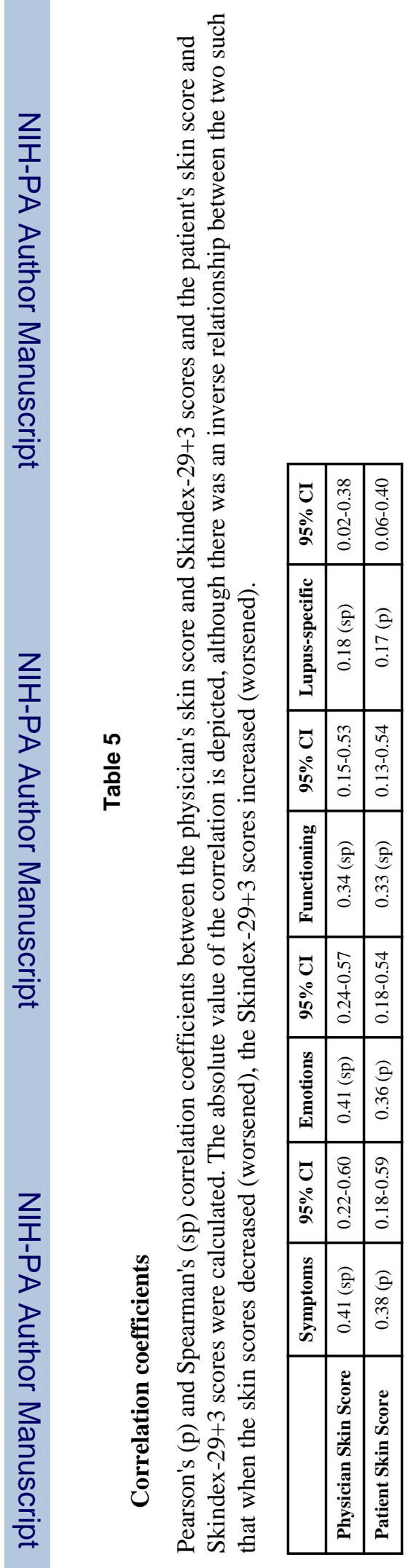

J Am Acad Dermatol. Author manuscript; available in PMC 2012 May 1. 